\title{
INVESTIGATING THE INFLUENCE OF COMMUNICATION AND INPUT DEVICES ON COLLABORATION IN VIRTUAL ENVIRONMENTS
}

\author{
Anastasiia Beznosyk, Chris Raymaekers, Karin Coninx, Peter Quax, Wim Lamotte \\ Hasselt University - $t U L-I B B T$, Expertise Centre for Digital Media \\ Wetenschapspark 2,B-3590 Diepenbeek (Belgium) \\ \{anastasiia.beznosyk, chris.raymaekers, karin.coninx, peter.quax, wim.lamotte\}@uhasselt.be
}

Keywords: Collaborative virtual environments, 3D manipulation, input devices.

Abstract: As collaborative virtual environments (CVEs) are becoming more popular for both entertainment and profes-
sional activities, it is important to know which factors influence the collaboration between participants. This
paper investigates two aspects of interaction in the context of collaboration between two users. A puzzle-
solving task in a basic interactive virtual environment was used as a case study. The influence of voice com-
munication was assessed in a user experiment. Furthermore, as different I/O devices can be used with this
kind of applications, the fact whether both participants are using the same device or not, was also checked.
From this study, we can conclude that the inclusion of voice communication is important when working on
the same task in a CVE, since this allows the participants to explicitly divide the work. On the other hand,
the usage of the same kind of device by both users does not significantly influence the collaboration. The
availability of different devices shows not to be a problem for the acceptance of CVEs, and therefore it is not
necessary to impose a certain device, which is obviously preferable, especially when dealing with home users.

\section{INTRODUCTION}

The necessity for communication and information sharing in virtual environments (VEs) caused the appearance of collaborative virtual environments (CVEs). Massive Multiplayer On-line Role Playing Games and virtual communities, such as Second Life (Linden Labs, http://www.secondlife.com, 2003), have contributed significantly to this success.

The variety of computational resources nowadays provides a wide range of possibilities for the developers of virtual worlds. The potential of these resources leads to the question of their effective combination into heterogeneous set-ups, in such a way that the tasks between participants can be divided according to their abilities. While single-user interaction has been studied at great lengths (Bowman et al., 2005), the problem of enabling detailed interaction using a heterogeneous set of input devices is non-trivial to solve. To achieve productivity and efficiency of collaboration a detailed investigation of different device combinations is needed.

Another aspect that has received little attention is the effect of communication while working in a 3D environment (Otto and Roberts, 2003; Ullah et al., 2009). Several studies have shown its importance to increase the degree of presence within the environment. These studies, however, do not take the productivity of the collaboration into account.

We have investigated the influence of heterogeneous interaction when collaborating while performing highly interactive tasks. More specifically, the experiment studies the influence of unequal conditions between participants, as they are collaborating using different input devices. Although the performance and feeling of collaboration can be considered as the task dependent characteristics we believe that the outcome obtained in this particular case study can be applied to a wider range of tightly coupled interactive tasks.

\section{RELATED WORK}

Most current CVEs support a high level of presence and immersion, however, the interaction is reduced to 
voice communication and common navigation. Detailed task performance has received less focus.

Some previous studies examined such topics as presence and co-presence (Schroeder et al., 2001). In other examples (Alhalabi and Horiguchi, 2001; Duval et al., 2006) collaboration was supported between 2 users with equal performance abilities. Another example of interaction, that is homogeneous with respect to the I/O devices, was presented by Pinho et al. (Pinho et al., 2008) but in this study responsibilities were divided between the participants so they were able to complete the task only by cooperating.

Performance, leadership and presence have been identified as key issues in virtual environments (Heldal et al., 2005). Some of these factors depend on the type of the virtual environment and the tasks to be performed, others on computational resources and available devices. For example, experiments (Steed et al., 1999) have proved that the person who had the highest degree of feeling to be present in the VE was singled out as the leader. In the meantime there was little research that investigated the influence of communication between participants on collaboration (Otto and Roberts, 2003). There is also a lack of studies investigating the absence of communication and possible negative reactions due to the ability to talk.

The contribution of our work is the combination and comparison of different setups of input devices. Although several studies are known where different devices were combined, they included similar kinds of devices (McLaughlin et al., 2003). We compare not only performance between different device types (relative and absolute) within a homogeneous setup, but we also show the heterogeneous performance when everyone is using a different device.

\section{EXPERIMENT}

In order to assess the influence of communication and the I/O devices used, a user experiment was conducted. Pairs of participants had to collaborate in a highly interactive puzzle-solving task. To investigate the effect of a homogeneous setup versus a heterogeneous setup, a SpaceMouse and a Phantom device were chosen. While the Phantom device has an absolute mapping of its position and orientation to the 3D cursor, the SpaceMouse is a relative device.

\subsection{Participants}

Six female and twenty-six male unpaid volunteers, ranging in age between 23 and 39 participated in the experiment. They were randomly divided into 16 pairs. All test persons had a computer science background. All but one participant were right-handed and used their dominant hand to operate the devices. Most participants had no experience working with the input devices involved. The others only had experience from participating in other user tests, where the same devices were involved.

\subsection{Apparatus}

For the experiment, two desktop computers were connected over a LAN. For output two 19" monitors were used. As indicated earlier, a Phantom device and a SpaceMouse were used for input. Participants were sitting in the same room quite close to each other but they were not able to see the partner's screen, as can be seen in figure 1 .

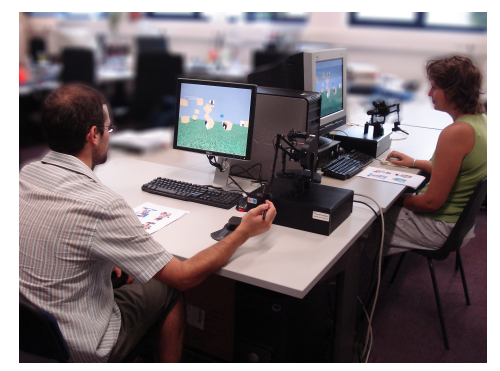

Figure 1: Experimental setup.

\subsection{Design}

For selection and manipulation within the 3D environment, the virtual hand technique (Bowman et al., 2005) was chosen. The input devices were represented by cones of different colors.

A between-subjects design was used for the presence or absence of voice communication. The device order was counterbalanced using a Latin square design. This resulted in four puzzles that had to be solved by each pair: two using homogeneous interaction and two with heterogeneous interaction. The dependent variables were the completion time for each group and the amount of correctly placed blocks for each participant.

\subsection{Procedure}

The puzzle-task consisted of collecting 12 pieces, representing a picture, where one was already provided as visual guide (see figure 2). Each session consisted of a new puzzle. The puzzles were assigned to all pairs in the same order and had the same level of complexity. The participants saw the completed picture in front of them during the experiment. Puzzle pieces were represented as cubes, with the picture on one 
face, dispersed in the 3D environment. Start and Finish buttons had to be clicked when they were ready to perform the task and as soon as the task had been completed. The selected puzzle piece was highlighted for both participants to avoid same cube selection.

In order to investigate the influence of communication, half of the pairs were instructed to talk to each other. As not all participants spoke the same language as first language, all communication was held in English. Although the participants were not native speakers, we believe this did not significantly influence the results as all of them were used to speak English on a regular basis.

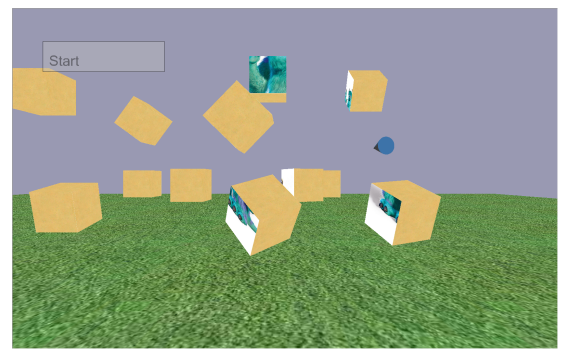

Figure 2: Experimental task in the VE.

In the beginning of the experiment, participants were allowed to practice with both devices. Once the training was completed, the four puzzle-solving sessions were performed. After each session, the participants had to fill in a questionnaire, based on (Steed et al., 1999).

\section{ANALYSIS AND RESULTS}

\subsection{Communication}

In order to assess the users' performance, we analyzed how the ability to talk influenced the total time to perform the task. Against our expectations the task was completed quicker in all 4 sessions for the pairs who were not allowed to talk. In table 1 the performance results of homogeneous (HM) and heterogeneous (HT) setups are compared (M - mean, SD standard deviation).

Table 1: Total time (sec).

\begin{tabular}{|l|c|r|c|c|}
\hline \multirow{2}{*}{} & \multicolumn{2}{|c|}{ Communication } & \multicolumn{2}{c|}{ No communication } \\
\cline { 2 - 5 } & $\mathrm{M}$ & $\mathrm{SD}$ & $\mathrm{M}$ & $\mathrm{SD}$ \\
\hline Homogeneous interaction & 206.01 & 89.43 & 182.30 & 64.97 \\
\hline Heterogeneous interaction & 235.64 & 133.75 & 166.75 & 58.50 \\
\hline
\end{tabular}

Total time on task was analyzed using a linear mixed model. We found a significant main effect of communication on total time $\left(F_{1,58}=4.768, p=\right.$
0.03). This indicates that people spent significantly more time on a task when they were able to talk to the partner. This occurred regardless of the devices used, as no interaction effect was found between the setup and communication.

One of the characteristics that test persons had to evaluate was the convenience with a certain device. Figure 3 shows that using the same device setup under different conditions changed the feeling of the participants. For example, when they were allowed to talk, the mean value of "convenience" was higher. This can be explained by the fact that when people were allowed to communicate they were able to ask the other person for help so they did not feel themselves being aside from the performance.

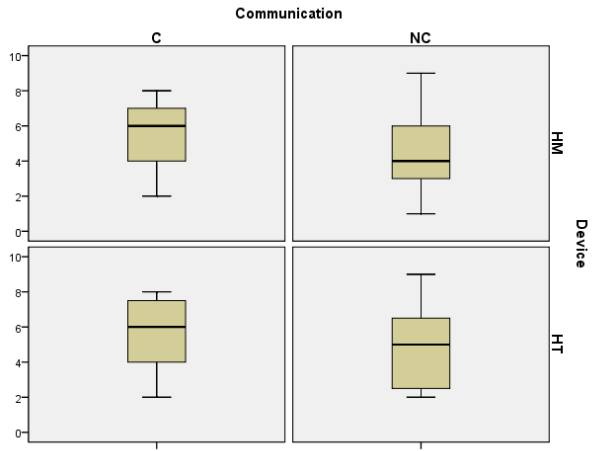

Figure 3: Convenience with the device, for heterogeneous (HT) and homogeneous (HM) setups.

The generalized linear model (GLM) analysis has shown that for the no-communication case there is a significant main effect of the convenience with device on the total amount of blocks placed by a participant $\left(\chi^{2}(1)=5.671, p=0.017\right)$. For the case where communication was allowed, there is a main effect of the device convenience on the total time $\left(F_{1,27}=11.094\right.$, $p=0.003$ ).

The results of the experiment show that the ability to communicate in a CVE increases the feeling of collaboration independent of the setup (see table 2).

Table 2: Feeling of collaboration.

\begin{tabular}{|c|c|c|c|c|}
\hline \multirow{2}{*}{} & \multicolumn{2}{|c|}{ Communication } & \multicolumn{2}{c|}{ No communication } \\
\cline { 2 - 5 } & $\mathrm{M}$ & $\mathrm{SD}$ & $\mathrm{M}$ & $\mathrm{SD}$ \\
\hline Feeling of collaboration & 7.19 & 1.36 & 5.59 & 2.27 \\
\hline
\end{tabular}

We therefore come to the following conclusion. When it is important to achieve a better feeling of "working together", communication plays an essential role. But if it is crucial to perform the task quicker and divide it between the participants, the ability to communicate can decrease the productivity and efficiency of the collaboration. 
For the sessions with communication, the linear mixed model analysis shows a main effect of the partner's share to the task solving on the "feeling of collaboration" $\left(F_{7,11}=4.327, p=0.015\right)$. We found that the contribution to the placing of the cubes is not significant for this case. The partner's convenience with the device has main effect on collaboration $\left(F_{9,20}=2.445, p=0.046\right)$, which indicates that people felt to collaborate significantly more when their partners were comfortable with the devices.

For the case where no conversation was allowed, the own contribution to the cube placing is significant for the level of collaboration $\left(F_{1,27}=13.099\right.$, $p=0.001)$.

Another relevant result that was obtained by comparison of the performance under two conditions shows that in all sessions the mean values of contribution to the task by the participants were higher when they had the chance to talk to each other and discuss the task (see figure 4).

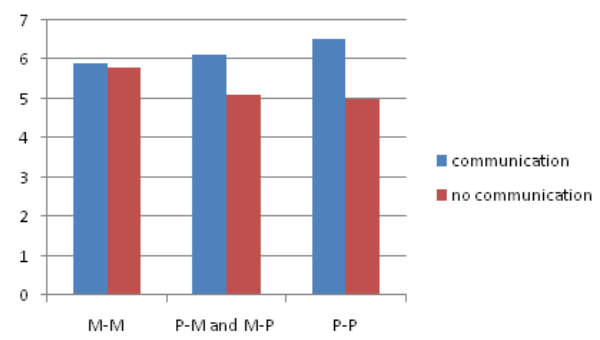

Figure 4: Contribution to the task, when using a SpaceMouse (M) or a Phantom device (P).

Amongst others we wanted to determine the factors that define leadership when collaborating. One of our initial expectations was that the person would be considered as a leader more when he/she performs more active and places more blocks to the correct position in both cases. But for the communication cases it turned out that people who were assessed as being talkative were seen as the leaders, rather than participants who performed more. Our analysis led to the conclusion that when people are not able to communicate with each other, they take the lead when they feel comfortable to operate within the environment and this can be achieved by using suitable devices.

Participants were also asked to estimate to what extent they considered themselves as a leader. Furthermore, we asked to evaluate the extent of the leadership of the partner. In our opinion it is interesting to compare how these evaluations differ from each other under different conditions.

In the cases where communication was allowed, the persons who operated a SpaceMouse had the same idea about their leadership as their partners. In contrast, when the person was using a Phantom device, he/she tended to overrate own leadership value comparing to the one given by their partner. For the cases without communication, there is little difference between the self evaluation and the partner's rating.

Comparing the correlation between leadership and convenience with the device under the two conditions the following can be observed: for the communication case the leadership is defined mostly by the communication, while for the no-communication cases, the comfort of device usage is more influential

The performed analysis has shown that the correlation between the device convenience and leadership is stronger for no-communication cases. For the communication cases, there is an input of the communication to the correlation.

Mixed model analysis has shown no main effect of the contribution characteristics on the leadership value for the communication case. Contrary to this, for no-communication cases, there is a significant main effect of blocks placed by the participant $\left(F_{1,24}=5.064, p=0.34\right)$ and his contribution to the task solving $\left(F_{1,24}=6.247, p=0.020\right)$ on the level of his perceived leadership.

\subsection{Homogeneous and Heterogeneous Setups}

Our research was organized with the aim to investigate the potential of unequal interaction (heterogeneous setup) within one collaborative environment. One of the main goals we wanted to achieve was to see the possibilities and drawbacks of creating heterogeneous collaborative environments where everyone can perform specific tasks to achieve a common aim. Although we did not perform such a separation in this experiment, results have shown that in communication cases, participants divided their roles by themselves.

Analyzing the heterogeneous and homogeneous setups, based on a linear mixed model, we found no significant difference for the performance time, but there is a main effect of convenience with the device, $F_{1.57}=4.050, p=0.049$ for participant $\# 1$ and $F_{1.57}$ $=23.224, p<0.001$ for participant \#2. There was no significant interaction effect between 2 devices. This result provides us with the ability to design collaborative environments where the use of certain devices does not limit other device types that could be involved.

Using a GLM analysis of the effect of different conditions on the total amount of blocks placed correctly, we found a main effect of one of the device types $\left(\chi^{2}(1)=3.869, p=0.049\right)$. This indicates that a pair placed significantly more blocks if at least one of 
the devices was comfortable to use.

The change from a homogeneous device combination to a heterogeneous one during communication session does not bring any significant result (Fig. 5). However, when no communication is present, there is a tendency to improve the feeling of collaboration and increase the efficiency in the heterogeneous setup (Fig. 6). Although the available activities of both participants were the same, they felt more comfortable when they could rely on their partner using a different device.

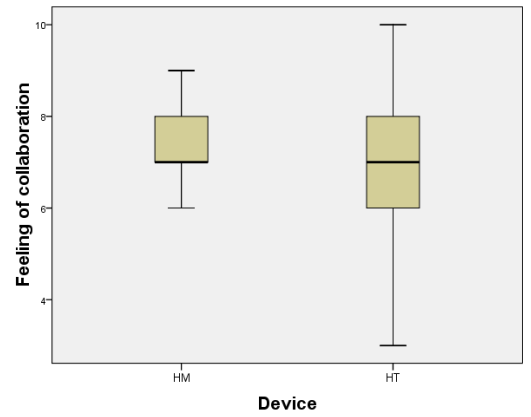

Figure 5: Effect of the device on the collaboration in the communication case.

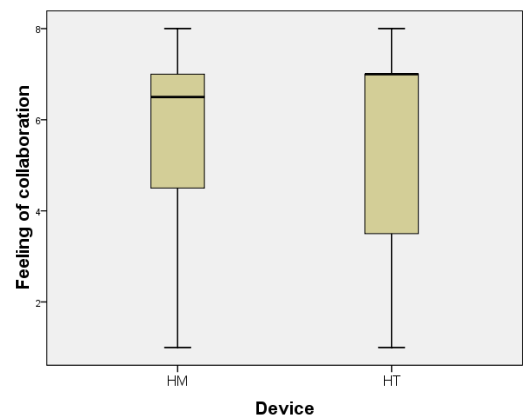

Figure 6: Effect of the device on the collaboration in the no-communication case.

According to the total time to perform the task, we can see that the heterogeneous setup reduces the completion time compared to the SpaceMouseSpaceMouse case. It takes, however, longer than the Phantom-Phantom case.

Figure 7 shows the dependence between the share in task solving and the type of the device used. Because the SpaceMouse was considered by most participants as a more complicated device to use, the SpaceMouse-SpaceMouse case is rather changing the character of the trend (jumping from min extreme to max several times). The homogeneous case with the Phantoms shows that the contribution is quite high and remains almost the same during the whole experiment. In the heterogeneous case, the participant using the Phantom has a higher share in the task

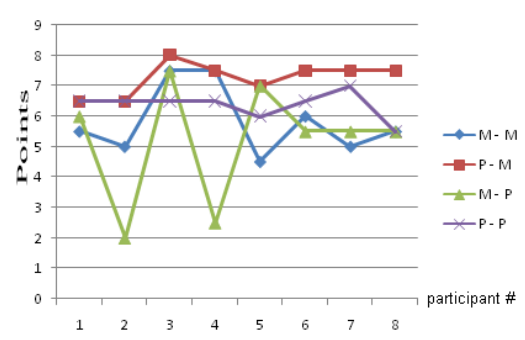

Figure 7: Share in task solving.

solving compared to the same device in the homogeneous case. The heterogeneous case SpaceMousePhantom shows that the participant using the SpaceMouse felt as having contributed less in solving the task. Analysis has shown that users with less comfortable devices did not enjoy the collaboration. They were not always able to follow their partners. This is the main drawback of unequal collaboration. Such consequences should orient the developers of collaborative environments towards more balanced cooperation. The development of appropriate tasks, where the diversity of abilities can be beneficial for all the participants and involve everyone at the same level of cooperation, is one possible solutions.

For this reason, it is also important to investigate the correlation between the device usage and the contribution to the block placing. Figure 8 shows this correlation for the cases, where communication was present.

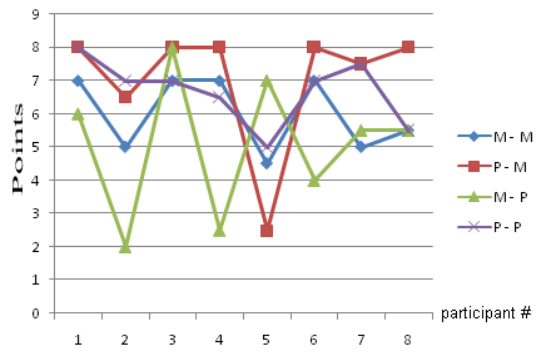

Figure 8: Block placing in the communication case.

As can be seen from the graph for the homogeneous case, most of the values remain within a certain range and have little data spread. For the heterogeneous sessions more extreme values can be observed, especially in the case when the participant was using the SpaceMouse. But it is also necessary to keep in mind that during the communication case the "placing cubes" variable did not always show the reality, as some pairs divided the work: one partner would orientate all puzzle pieces, while the other would place them in the puzzle. That is why we also 
present the same analysis for "placing cubes" in the no-communication case (see figure 9).

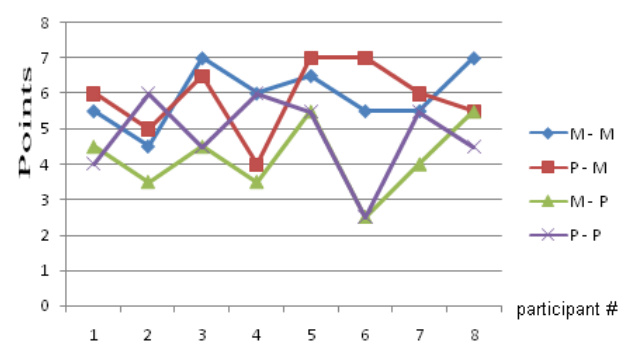

Figure 9: Block placing within no-communication case.

The graph of no-communication case shows a more balanced trend with less extreme values. There is also not much data spread for the homogeneous case which was caused by more balanced collaboration.

The results obtained in this comparative study of homogeneous and heterogeneous setups can be used in 2 ways. The first conclusion that can be drawn here is that unequal interaction (in terms of $\mathrm{I} / \mathrm{O}$ devices) can decrease the efficiency and contribution of one of the participants, and cause an uncomfortable experience for these participants in a CVE. However as, in reality, the device that is most suited for performing a task is not always available, we can conclude that a heterogeneous setup still allows users to work in an efficient manner. On the other hand, we can think about other types of tasks to be performed in a CVE, where participants have limited abilities and should perform different tasks to achieve the common goal.

\section{Conclusions}

The results obtained in this case study have an important impact for the implementation of collaborative virtual environments. One implication is that inclusion of communication is important when working together in a virtual environment, since this allows the participants to explicitly divide the work. Communication also increases the feeling of collaborating together but at the same moment decreases the performance. Another important result is that the combination of different devices does not significantly influence the collaboration as no interaction effect of the devices that can be combined was found. To increase the efficiency of heterogeneous collaboration we can think of other activities to be performed in the VE where every participant can supplement each other performance. Of course further research is necessary to explore other aspects of successful collaboration.

\section{ACKNOWLEDGEMENTS}

The research described in this paper is directly funded by Hasselt University through the BOF framework. The authors would like to thank Lode Vanacken for his assistance with the implementation and Roel Braeken of the UHasselt Center for Statistics for helping to choose the statistical tests.

\section{REFERENCES}

Alhalabi, M. O. and Horiguchi, S. (2001). Tele-handshake: Cooperative shared haptic virtual environment. Research report, pages 1-12.

Bowman, D. A., Kruijff, E., LaViola, J. J., and Poupyrev, I. (2005). 3D User Interfaces, Theory and Practice. Addison-Wesley.

Duval, T., Lecuyer, A., and Thomas, S. (2006). Skewer: a $3 \mathrm{~d}$ interaction technique for 2-user collaborative manipulation of objects in virtual environments. In $3 D U I$ '06: Proceedings of the 3D User Interfaces, pages 6972, Washington, DC, USA. IEEE Computer Society.

Heldal, I., Steed, A., and Schroeder, R. (2005). Evaluating collaboration in distributed virtual environments for a puzzle-solving task. In Proceedings of the 11th International Conference on HCI.

Linden Labs, http://www.secondlife.com (2003).

McLaughlin, M., Sukhatme, G., Peng, W., Zhu, W., and Parks, J. (2003). Performance and co-presence in heterogeneous haptic collaboration. Haptic Interfaces for Virtual Environment and Teleoperator Systems, International Symposium on, pages 285 - 291.

Otto, O. and Roberts, D. (2003). Importance of communication influences on a highly collaborative task. Distributed Simulation and Real-Time Applications, IEEE International Symposium on, page 195.

Pinho, M. S., Bowman, D. A., and Freitas, C. M. (2008). Cooperative object manipulation in collaborative virtual environments. In J. Braz. Comp. Soc., volume 14, pages 54-67.

Schroeder, R., Steed, A., Axelsson, A.-S., Heldal, I., Åsa Abelin, Wideström, J., Nilsson, A., and Slater, M. (2001). Collaborating in networked immersive spaces: as good as being there together? Computers \& Graphics, 25(5):781 - 788 .

Steed, A., Slater, M., Sadagic, A., Bullock, A., and Tromp, J. (1999). Leadership and collaboration in shared virtual environments. In $V R$ '99: Proceedings of the IEEE Virtual Reality, page 112, Washington, DC, USA. IEEE Computer Society.

Ullah, S., Otmane, S., Richard, P., and Mallem, M. (2009). Human performance in cooperative virtual environments : the effect of visual aids and oral communication. IJVR, 8(4):79-86. 